PROCEEDINGS OF THE

AMERICAN MATHEMATICAL SOCIETY

Volume 34, Number 2, August 1972

\title{
THE MORSE LEMMA ON BANACH SPACES
}

\author{
A. J. TROMBA
}

Abstract. Let $f: U \rightarrow R$ be a $C^{3}$ map of an open subset $U$ of a Banach space $E$. Let $p \in U$ be a critical point of $f\left(d f_{p}=0\right)$. If $E$ is a conjugate space $\left(E=F^{*}\right)$ we define what it means for $p$ to be nondegenerate. In this case there is a diffeomorphism $\gamma$ of a neighborhood of $p$ with a neighborhood of $0 \in E, \gamma(p)=0$ with

$$
f \circ \gamma^{-1}(x)=\frac{1}{2} d^{2} f_{p}(x, x)+f(p) .
$$

In this paper we define a notion of nondegeneracy for critical points of smooth real valued functions defined on open subsets of a Banach space $E$ which is a dual space, and we shall prove the Morse lemma with our definition. Earlier notions of nondegeneracy which implied the Morse lemma are stronger than ours and in particular had the unfortunate consequence of implying that $E \approx E^{*}$. See Palais [3] and [4] for the case $E=$ Hilbert space and $E \approx E^{*}$ respectively.

Definition. Let $E_{0}$ be a Banach space and let $E=E_{0}^{*}$ be the dual space of $E_{0}$. Denote by $\langle\rangle:, E \times E_{0}$ the bilinear pairing of $E_{0}$ and $E$ given by $\langle f, x\rangle=f(x)$.

In the paragraphs below we shall not be too careful about the order of the terms in $\langle$,$\rangle ; e.g., \langle x, f\rangle$ instead of $\langle f, x\rangle$ and no confusion should arise from this.

A continuous linear map $A \in L\left(E, E_{0}\right)$ is said to be symmetric if $\langle y, A x\rangle=\langle x, A y\rangle$ for all $x, y \in E$. We note this by writing $A \in L_{s}\left(E, E_{0}\right)$. The proof of the following is standard.

Lemma 1. If $A \in L_{s}\left(E, E_{0}\right)$ is injective then $A(E)$ is dense in $E_{0}$.

DEFINITION. Let $U \subset E$ be an open subset with $f: U \rightarrow R C^{2}$-differentiable. We say that $f$ is weak (*) smooth if for each fixed $t \in U$ and $h \in E$ the linear functional on $E$ given by $k \rightarrow d^{2} f_{t}(h, k)$ is continuous in the weak $\left.{ }^{*}\right)$ topology of $E$ induced by $E_{0}$. If $E$ is reflexive every $C^{2}$-map on $U$ is weak $(*)$ smooth.

LEMMA 2. Let $t \in U$ be fixed with $f: U \rightarrow R C^{2}$ and weak (*) smooth. Then $d^{2} f_{t}$ induces a map $A_{t} \in L_{s}\left(E, E_{0}\right)$ with $k\left(A_{t}(h)\right)=\left\langle k, A_{t}(h)\right\rangle=$ $d^{2} f_{t}(h, k)$.

Received by the editors February 16, 1971.

AMS 1970 subject classifications. Primary 49F15, 58E05; Secondary 58C15. 
Proof. Since $k \rightarrow d^{2} f_{t}(h, k)$ is continuous in the weak $(*)$ topology it is given by an element of $E_{0}$, say $A_{t}(h)$ according to the rule $k\left(A_{t}(h)\right)=$ $d^{2} f_{t}(h, k)$. (For a proof of this fact see $\left[1\right.$, p. 421].) $A_{t}$ is readily seen to be linear in $h$ since $d^{2} f_{t}(h, k)$ is. This completes the proof.

Let $U$ be a convex neighborhood of a critical point $p$ of $f: U \rightarrow R$ (i.e., $d f_{p}=0$ ) which we assume to be $C^{3}$ and weak (*) smooth. Then by Taylor's formula for $x \in U, x=p+\xi$,

$$
f(p+\xi)=f(p)+\int_{0}^{1}(1-\lambda) d^{2} f(p+\lambda \xi)(\xi, \xi) d \lambda .
$$

Let $B_{x}: E \times E \rightarrow R$ be the symmetric bilinear form given by

$$
B_{x}(h, k)=\int_{0}^{1}(1-\lambda) d^{2} f(p+\lambda \xi)(h, k) d \lambda .
$$

Then, by the above remarks, $B_{x}(h, k)=\left\langle k, A_{x} h\right\rangle$ where $A_{x} \in L_{s}\left(E, E_{0}\right)$. $A: U \rightarrow L_{s}\left(E, E_{0}\right)$ is called the bilinear representative of $f$ in $U$. If $f$ is $C^{3}$ then $A$ is $C^{\mathbf{1}}$. If $p=0$ the above Taylor expansion for $f$ can then be written

$$
f(x)=B_{x}(x, x)+f(0)=\left\langle A_{x}(x), x\right\rangle+f(0)
$$

for $x$ near 0 . In the following paragraphs we shall assume for simplicity that our critical point $p$ is in fact 0 .

Definition. The critical point $p$ is nondegenerate if there is a neighborhood $W \subset U$ and positive constants $C_{1}$ and $C_{2}$ with

(1) $A_{0}$ injective,

(2) $\left\|D A_{t}(h)(y)\right\| \leqq C_{1}\|h\|\left\|A_{t^{\prime}} y\right\|$,

(3) $\left\|D A_{t_{1}}(h)(y)-D A_{t_{2}}(h)(y)\right\| \leqq C_{2}\|h\| \cdot\left\|t_{1}-t_{2}\right\| \cdot\left\|A_{t^{\prime}} y\right\|$,

for all $t, t^{\prime}, t_{1}, t_{2} \in W$, where $D A_{t}$ denotes the Fréchet derivative of $A$ with respect to the subscript variable.

REMARK. In the case $E=H$, Hilbert space, considered by Palais and Smale, $A_{0} \in L_{s}(H, H)$ was required to be an isomorphism for $p$ to be a nondegenerate critical point of $f$. The requirement that $A_{0}$ be an isomorphism implies (1), (2), and (3), and so these conditions are weaker than the nondegeneracy condition of Palais and Smale.

Lemma 3. Let $A$ be a bilinear representative off at $p$. Then there exists a neighborhood $W$ of 0 and constant $K>1$ with the property that

$$
K^{-1}\left\|A_{0} y\right\| \leqq\left\|A_{t} y\right\| \leqq K\left\|A_{0} y\right\| .
$$

Proof. By the mean value theorem we have

$$
A_{t} y-A_{0} y=\int_{0}^{1} D A_{\lambda t}(t)(y) d \lambda
$$


Let $W$ be a bounded convex neighborhood of $0 \in E$ where the nondegeneracy conditions hold. Then

$$
\left\|A_{t}-A_{0} y\right\| \leqq \int_{0}^{1}\left\|D A_{\lambda, t}(t)(y)\right\| d \lambda \leqq C_{1}\|t\| \cdot\left\|A_{t^{\prime}} y\right\|
$$

for all $t, t^{\prime} \in W$.

From this (dividing by $\left\|A_{t^{\prime}} y\right\|$ ), we can conclude that

$$
\left|\frac{\left\|A_{t} y\right\|}{\left\|A_{t^{\prime}} y\right\|}-\frac{\left\|A_{0} y\right\|}{\left\|A_{t^{\prime}} y\right\|}\right| \leqq C_{1}\|t\| .
$$

Then setting $t^{\prime}=0$ we get $\left\|A_{t} y\right\| \leqq\left(1+C_{1}\|t\|\right)\left\|A_{0} y\right\|$ and setting $t^{\prime}=t$ we get $\left\|A_{0} y\right\| \leqq\left(1+C_{1}\|t\|\right)\left\|A_{t} y\right\|$. Finally, setting $K=1+\sup _{t \in W} C_{1}\|t\|$ concludes the proof.

REMARK. The above lemma implies that the map $A: W \rightarrow L_{s}\left(E, E_{0}\right)$ takes values $A_{t}$ which are injective maps for all $t \in W$.

Lemma 4. Let $A$ be a bilinear representative off at $p$. Then there exists a neighborhood $W$ of 0 and constants $C_{1}$ and $C_{2}$ with

$$
\left\|A_{t_{1}} y-A_{t_{2}} y\right\| \leqq C_{1}\left\|t_{1}-t_{2}\right\| \cdot\left\|A_{t^{\prime}} y\right\|
$$

for $t_{1}, t_{2}, t^{\prime} \in W$.

Proof. Follows directly from the mean value theorem.

LEMMA 5. Let $f: \mathcal{O} \rightarrow R$ be a real valued $C^{3}$ weak $\left({ }^{*}\right)$ smooth function defined in the neighborhood $\mathcal{O}$ of the origin of $E$. Suppose 0 is a nondegenerate critical point of $f$. From before

$$
f(x)=\left\langle A_{x}(x), x\right\rangle+f(0) .
$$

Then there exists a $C^{1}$ map $t \rightarrow Q_{t} \in G L(E)$, the general linear group of $E$ with $A_{t} Q_{t}=A_{0}$ and $Q_{0}=I$.

Proof. Let $W$ be a convex neighborhood of 0 so that Lemmas 3 and 4 hold. Let $t \in W$ be fixed. Then by the remark after Lemma $3, A_{t}$ is injective; moreover, Range $A_{t}$ is dense in $E_{0}$. Let $y \in E$. Define $Q_{t}(y) \in E_{0}^{*}$ by

$$
\left\langle Q_{t}(y), A_{t}(x)\right\rangle=Q_{t}(y)\left(A_{t} x\right)=\left\langle y, A_{0} x\right\rangle .
$$

By Lemma $3, Q_{t}(y)$ is continuous. Since Range $A_{t}$ is dense this defines $Q_{t}(y)$ on all of $E_{0}$. It is easy to check that for each $t \in W$, that $Q_{t}$ is linear. We shall show that $Q_{t}$ is an isomorphism and that the map $t \rightarrow Q_{t}$ is $C^{\mathbf{1}}$.

First, since $A_{t}$ is symmetric for each $t \in W,\left\langle A_{t} Q_{t} y, x\right\rangle=\left\langle A_{0} y, x\right\rangle$. Therefore,

$$
\left|\left\langle A_{t} x, Q_{t} y\right\rangle\right|=\left|\left\langle A_{t} Q_{t} y, x\right\rangle\right|=\left|\left\langle A_{0} x, y\right\rangle\right| \leqq K\|y\| \cdot\left\|A_{t} x\right\| .
$$


Thus,

$$
\left|\left\langle Q_{t} y, A_{t} x /\left\|A_{t} x\right\|\right\rangle\right| \leqq K\|y\|
$$

for all $x \in E$. This implies that $\left\|Q_{t} y\right\| \leqq K\|y\|$. (Recall for $f \in E_{0}^{*},\|f\|=$ $\sup _{\|x\|=1}|\langle f, x\rangle|$ and if $S \subset E_{0}$ is dense $\|f\|=\sup _{\|x\|=1 ; x \in S}|\langle f, x\rangle|$.) Secondly,

$$
\left|\left\langle A_{0} x, y\right\rangle\right|=\left|\left\langle A_{0} y, x\right\rangle\right|=\left|\left\langle A_{t} Q_{t} y, x\right\rangle\right| \leqq\left\|Q_{t} y\right\| \cdot\left\|A_{t} x\right\| \leqq K\left\|Q_{t} y\right\| \cdot\left\|A_{0} x\right\| .
$$

Thus

$$
\left|\left\langle A_{0} x /\left\|A_{0} x\right\|, y\right\rangle\right| \leqq K\left\|Q_{t} y\right\|
$$

for all $x, y \in E$, which implies that $\left\|Q_{t} y\right\| \geqq K^{-1}\|y\|$, which further implies that for each $t \in W, Q_{t}$ is injective, continuous and has closed range. To see that $Q_{t}$ is invertible, let $y \in E$ be arbitrary. Define $P_{t}(y) \in E_{0}^{*}$ by $P_{t}(y)\left(A_{0} x\right)=\left\langle A_{t} x, y\right\rangle$. Then

$$
\left\langle A_{0} P_{t} y, x\right\rangle=\left\langle A_{t} y, x\right\rangle .
$$

As before, $P^{t}$ will be continuous, injective and have closed range. Now

$$
\left\langle A_{t} Q_{t} P_{t} y, x\right\rangle=\left\langle A_{0} P_{t} y, x\right\rangle=\left\langle A_{t} y, x\right\rangle,
$$

for all $x, y$. This implies that $A_{t} Q_{t} P_{t}=A_{t}$ and since $A_{t}$ is injective, we have that $Q_{t} P_{t}=I$, which shows that $Q_{t}$ is onto, and hence invertible for each $t$.

We must now show that the map $t \rightarrow Q_{t}$ is $C^{1}$. The continuity of this map is guaranteed by Lemma 4. For

Thus

$$
\begin{aligned}
\left|\left\langle Q_{t_{1}} y-Q_{t_{2}} y, A_{t_{1}} x\right\rangle\right| & =\left|\left\langle Q_{t_{1}} y, A_{t_{1}} x\right\rangle-\left\langle Q_{t_{2}} y, A_{t_{1}} x\right\rangle\right| \\
& =\left|\left\langle A_{0} y, x\right\rangle-\left\langle Q_{t_{2}} y, A_{t_{1}} y\right\rangle\right| \\
& =\left|\left\langle Q_{t_{2}} y, A_{t_{2}} x\right\rangle-\left\langle Q_{t_{2}} y, A_{t_{1}} x\right\rangle\right| \\
& =\left|\left\langle Q_{t_{2}} y, A_{t_{2}} x-A_{t_{1}} x\right\rangle\right| \leqq\left\|Q_{t_{2}} y\right\| \cdot\left\|A_{t_{2}} x-A_{t_{1}} x\right\| \\
& \leqq K C_{1}\|y\| \cdot\left\|t_{1}-t_{2}\right\| \cdot\left\|A_{t_{1}} x\right\| .
\end{aligned}
$$

or

$$
\left\|Q_{t_{1}} y-Q_{t_{2}} y\right\| \leqq C_{1} K\|y\| \cdot\left\|t_{1}-t_{2}\right\|
$$

$$
\left\|Q_{t_{1}}-Q_{t_{2}}\right\| \leqq C_{1} K\left\|t_{1}-t_{2}\right\|
$$

which gives the continuity of the map $t \rightarrow Q_{t}$.

To see that the map is smooth, we use the same sort of trick we have already employed. Our candidate for the derivative of $Q_{t}$ will be the map defined by

$$
\left\langle D Q_{t}(h)(y), A_{t} x\right\rangle=-\left\langle D A_{t}(h)(x), Q_{t} y\right\rangle .
$$

The nondegeneracy assumption assures us that the map $D Q_{t}$ is well defined and continuous. 
Let $t_{0} \in W$ be fixed and let $h$ be so small that $t_{0}+h \in W$. Then

$$
\begin{aligned}
\left\langle Q_{t_{0}+h} y-Q_{t_{0}} y, A_{t_{0}} x\right\rangle & =\left\langle Q_{t_{0}+h} y, A_{t_{0}} x\right\rangle-\left\langle Q_{t_{0}} y, A_{t_{0}} x\right\rangle \\
& =\left\langle Q_{t_{0}+h} y, A_{t_{0}} x-A_{t_{0}+h} x\right\rangle .
\end{aligned}
$$

By the Fréchet differentiability of $t \rightarrow A_{t}$ this is equal to

where

$$
\left\langle Q_{t_{0}+h} y,-D A_{t_{0}}(h)(x)-R_{t_{0}}(h)(x)\right\rangle
$$

and

$$
A_{t_{0}+h}-A_{t_{0}}=D A_{t_{0}}(h)+R_{t_{0}}(h)
$$

$$
R_{t_{0}}(h)(x)=\int_{0}^{1}\left[D A_{t_{0}+\lambda h}(h)(x)-D A_{t_{0}}(h)(x)\right] d \lambda .
$$

(This implies that $\left\|R_{t_{0}}(h)(x)\right\| \leqq C_{2}\|h\|^{2} \cdot\left\|A_{t_{0}} x\right\|$.) Continuing the string of equalities we get $(*)$ equal to

$$
\begin{aligned}
\left\langle Q_{t_{0}+h}(y),-\right. & \left.D A_{t_{0}}(h)(x)\right\rangle-\left\langle Q_{t_{0}+h}(y), R_{t_{0}}(h)(x)\right\rangle \\
= & -\left\langle Q_{t_{0}}(y), D A_{t_{0}}(h)(x)\right\rangle+\left\langle Q_{t_{0}+h}(y)-Q_{t_{0}}(y),-D A_{t_{0}}(h)(x)\right\rangle \\
& -\left\langle Q_{t_{0}+h}(y), R_{t_{0}}(h)(x)\right\rangle \\
= & \left\langle D Q_{t_{0}}(h)(y), A_{t_{0}} x\right\rangle+\left\langle Q_{t_{0}+h}(y)-Q_{t_{0}}(y),-D A_{t_{0}}(h)(x)\right\rangle \\
& -\left\langle Q_{t_{0}+h}(y), R_{t_{0}}(h)(x)\right\rangle .
\end{aligned}
$$

Therefore

$$
\begin{aligned}
\mid\left\langle Q_{t_{0}+h} y-\right. & \left.Q_{t_{0}} y-D Q_{t_{0}}(h)(y), A_{t_{0}}(x)\right\rangle \mid \\
& \leqq\left\langle\left\langle Q_{t_{0}+h}(y)-Q_{t_{0}}(y), D A_{t_{0}}(h)(x)\right\rangle|+|\left\langle Q_{t_{0}+h}(y), R_{t_{0}}(h)(x)\right\rangle\right| .
\end{aligned}
$$

By the nondegeneracy conditions, we get that these two terms are bounded by

$$
\begin{aligned}
& \leqq\left\{C_{1} K\|h\| \cdot\|y\|\right\} \cdot\left\{\left\|D A_{t_{9}}(h)(x)\right\|\right\}+\{K\|y\|\} \cdot\left\{C_{2}\|h\|^{2}\left\|A_{t_{0}} x\right\|\right\} \\
& \leqq\left\{C_{1} K C_{2}+K C_{2}\right\}\|h\|^{2}\|y\| \cdot\left\|A_{t_{0}} x\right\| .
\end{aligned}
$$

Thus

$$
\left\|Q_{t_{0}+h} y-Q_{t_{0}} y-D Q_{t_{0}}(h)(y)\right\| \leqq\left\{C_{1} C_{2} K+K C_{2}\right\}\|h\|^{2}\|y\|
$$

which implies that

$$
\left\|Q_{t_{0}+h}-Q_{t_{0}}-D Q_{t_{0}}(h)\right\| \leqq\left\{C_{1} C_{2} K+K C_{2}\right\}\|h\|^{2} .
$$

Therefore

$$
\lim _{\|h\| \rightarrow 0} \frac{1}{\|h\|}\left\{\left\|Q_{t_{0}+h}-Q_{t_{0}}-D Q_{t_{0}}(h)\right\|\right\}=0
$$

and hence $t \rightarrow Q_{t}$ is Fréchet differentiable. 
The last thing to show is that $t \rightarrow D Q_{t}$ is continuous. Now

$$
\begin{aligned}
\left\langle D Q_{t_{1}}\right. & \left.(h)(y)-D Q_{t_{2}}(h)(y), A_{t_{1}} x\right\rangle \\
= & -\left\langle Q_{t_{1}} y, D A_{t_{1}}(h)(x)\right\rangle-\left\langle D Q_{t_{2}}(h)(y), A_{t_{1}} x\right\rangle \\
= & -\left\langle Q_{t_{1}} y, D A_{t_{1}}(h)(x)\right\rangle-\left\langle D Q_{t_{2}}(h)(y), A_{t_{2}} x\right\rangle \\
& \quad+\left\langle D Q_{t_{2}}(h)(y), A_{t_{2}} x-A_{t_{1}} x\right\rangle \\
= & -\left\langle Q_{t_{1}} y, D A_{t_{1}}(h)(x)-D A_{t_{2}}(h)(x)\right\rangle+\left\langle D Q_{t_{2}}(h)(y), A_{t_{2}} x-A_{t_{1}} x\right\rangle .
\end{aligned}
$$

As before

$$
\begin{aligned}
\mid\left\langle D Q_{t_{1}}(h)(y)-\right. & \left.D Q_{t_{2}}(h)(y), A_{t_{1}}(x)\right\rangle \mid \\
\leqq & \{K\|y\|\}\left\{C_{2}\|h\| \cdot\left\|t_{1}-t_{2}\right\| \cdot\left\|A_{t_{1}} x\right\|\right\} \\
& +\left\{\left\|D Q_{t_{2}}(h)(y)\right\| \cdot\left[C_{1}\right] \cdot\left\|t_{1}-t_{2}\right\| \cdot\left\|A_{t_{1}} x\right\|\right\} \\
\leqq & \left\{K C_{2}+K C_{2} C_{1}\right\}\left\{\|h\| \cdot\|y\| \cdot\left\|t_{1}-t_{2}\right\| \cdot\left\|A_{t_{1}} x\right\|\right\}
\end{aligned}
$$

which implies that

$$
\left\|D Q_{t_{1}}-D Q_{t_{2}}\right\| \leqq\left\{K C_{2}+K C_{2} C_{1}\right\}\left\|t_{1}-t_{2}\right\|
$$

which concludes the proof of Lemma 5.

TheOREM (Morse LeMma). Let $f: \mathcal{O} \rightarrow R$ be a $C^{3}$ and weak (*) smooth map with $0 \in \mathcal{O}$ a nondegenerate critical point of $f$. Then there exists a local $C^{1}$ diffeomorphism $\psi$ preserving the origin with

$$
f \circ \psi(x)=\frac{1}{2} d^{2} f_{0}(x, x)+f(0)=\left\langle A_{0} x, x\right\rangle+f(0) .
$$

Proof (following Palais [3]). By Lemma 5 here is a $C^{\mathbf{1}}$ map $t \mapsto$ $Q_{t} \in \mathrm{GL}(E)$ with $A_{t} Q_{t}=A_{0}$. Thus $Q_{t}^{*} A_{t}^{*}=A_{0}^{*}$ where $Q_{t}^{*} \in \mathrm{GL}\left(E^{*}\right)$. Since $A_{0}$ is symmetric, $\left(A_{0}^{*} x\right) y=x\left(A_{0} y\right)=y\left(A_{0} x\right)=\left(i A_{0} x\right) y$ for all $x, y \in E$, where $i: E_{0} \rightarrow E^{*}$ is the natural inclusion. Thus $A_{0}^{*}=i A_{0}$. Therefore

$$
Q_{t}^{*} A_{t}^{*}=i A_{t} Q_{t} \text {. }
$$

Also, $Q_{0}=I$ and thus, by the Taylor expansion for the squre root, $Q_{t}$ has a $C^{1}$ square root $S_{t}$ in some neighborhood $V_{0}$ of the origin. Now since equation $\left(\begin{array}{c}* \\ *\end{array}\right)$ is satisfied by $S_{t}$ (in fact by a polynomial in $Q_{t}$ or a limit of such), we have $S_{t}^{*} A_{t}^{*}=i A_{t} S_{t}$. Hence $S_{t}^{*} A_{t}^{*} S_{t}=i A_{t} S_{t}^{2}=i A_{0}$ and consequently

$$
A_{t}^{*}=R_{t}^{*}\left(i A_{0}\right) R_{t}
$$

where $R_{t}=S_{t}^{-1}$. From the bilinear representation of $f$ we have

$$
\begin{aligned}
f(x)-f(0) & =\left\langle A_{x}(x), x\right\rangle=x\left(A_{x}(x)\right)=\left(A_{x}^{*}(x)\right)(x)=\left(R_{x}^{*} i A_{0} R_{x}(x)\right)(x) \\
& =\left(i A_{0} R_{x}(x)\right)\left(R_{x} x\right)=R_{x}(x)\left(A_{0} R_{x}(x)\right)=\left\langle R_{x}(x), A_{0} R_{x}(x)\right\rangle .
\end{aligned}
$$

Let $\varphi(x)=R_{x}(x)$. Then $D \varphi_{0}(h)=R_{0}(h)=h$, since $Q_{0}=S_{0}=R_{0}=I$. Thus by 
the inverse function theorem, $\varphi$ has a local inverse $\psi$ restricted to a subneighborhood $V \subset V_{0}$. The map $\psi$ clearly satisfies the requirements of the lemma.

REMARK. If we require the map $\psi$ above to be only a local homeomorphism we can relax the nondegeneracy conditions to the following. Let $f(x)=\left\langle x, A_{x}(x)\right\rangle+f(p), A: U \rightarrow L_{s}\left(E, E_{0}\right)$ the bilinear representative of $f$. Then $p$ is nondegenerate if

(1') $A_{0}$ is injective, and there exists a subneighborhood $W \subset U$ of $p$ and constant $C$ so that

(2') $\left\|A_{t_{1}} y-A_{t_{2}} y\right\| \leqq C\left\|t_{1}-t_{2}\right\| A_{t^{\prime}} y \|$ for any $t_{1}, t_{2}, t^{\prime} \in W$.

We can define the notion of nondegenerate critical point of a $C^{3}$-map $f: M \rightarrow R$ on a $C^{3}$ Banach manifold $M$ modelled on $E=E_{0}^{*}$. We say that a critical point $p$ is nondegenerate if there is a chart $(\varphi, U)$ about $p, \varphi(U)=$ $\mathcal{O} \subset E$ with the property that $f \circ \varphi^{-1}: \mathcal{O} \rightarrow R$ is weak $\left({ }^{*}\right)$ smooth and has $\varphi(p)$ as a nondegenerate critical point.

From the last theorem we have

THEOREM. Let $f: M \rightarrow R$ be $C^{3}$ with $M$ modelled on $E=E_{0}^{*}$ and $p$ a nondegenerate critical point. Then there exists a local diffeomorphism $\gamma$ of a neighborhood of $\varphi(p)$ with a neighborhood of $0 \in E, \gamma(\varphi(p))=0$ and with

$$
f \circ\left(\varphi^{-1} \circ \gamma\right)(x)=d^{2}\left(f \circ \varphi^{-1}\right)_{\varphi(p)}(x, x)+f(p) .
$$

COROLlaRY. Nondegenerate critical points are isolated.

REMARK. It does not seem that this definition of nondegeneracy is independent of the choice of coordinate chart and hence does not appear to be a natural geometric notion of nondegeneracy for spaces $E$ which are not isomorphic to $E^{*}$. The author is at the moment unaware of a modification of nondegeneracy for general Banach manifolds, i.e., one which is independent of the selection of coordinate chart.

\section{BIBLIOGRAPHY}

1. N. Dunford and J. T. Schwartz, Linear operators. I. General theory, Pure and Appl. Math., vol. 7, Interscience, New York, 1958. MR 22 \#8302.

2. S. Lang, Introduction to differentiable manifolds, Interscience, New York, 1962. MR 27 \#5192.

3. R. Palais, Morse theory on Hilbert manifolds, Topology 2 (1963), 299-340. MR 28 \#1633.

4. —- The Morse lemma for Banach spaces, Bull. Amer. Math. Soc. 75 (1969), 968-971.

Department of Mathematics, University of California, Santa Cruz, California 95060 